\title{
Lateral sinus floor elevation performed with trapezoidal and triangular flap designs: Post operative Pain Measurement through thermal infrared imaging. A \\ randomized pilot study.
}

Antonio Scarano ${ }^{1}$, Felice Lorusso ${ }^{2}$, Arcangelo Merla ${ }^{3}$, Camillo D’Arcangelo ${ }^{4}$, and Pablo Santos de Oliveira $^{5}$,

1) Full Professor of Oral Surgery, Department of Medical, Oral and Biotechnological Sciences and CeSiMet, 'G. D’Annunzio' University of Chieti-Pescara, Via dei Vestini 31, 66100 Chieti-Italy, ascarano@unich.it;

2) PhD Student, Department of Medical, Oral and Biotechnological Sciences University of ChietiPescara, Via dei Vestini 31, 66100 Chieti- Italy, drlorussofelice@gmail.com;

3) Associate Professor Department of Neuroscience, Imaging and Clinical Sciences, University "G. d'Annunzio" - Chieti-Pescara, Via dei Vestini 31,66100 Chieti-Italy, arcangelo.merla@unich.it;

4) Full Professor, Department of Medical, Oral and Biotechnological Sciences and CeSi-Met, 'G. D'Annunzio' University of Chieti-Pescara, Via dei Vestini 31, 66100 Chieti-Italy, camillo.darcangelo@unich.it;

5) Associate Professor Department of Oral Implantology, Dental Research Division, College Ingà, UNINGÁ, 29312 Cachoeiro de Itapemirim- ES, Brazil, psoliveiraodonto@yahoo.com.br

Corresponding author:

Prof. Antonio Scarano, M.D., D.D.S.

Department of Medical, Oral and Biotechnological Sciences and CeSi-Met

Via dei Vestini 31, 66100 Chieti (Italy)

Fax: +39-0871-3554076

E-mail:ascarano@unich.it 


\begin{abstract}
Background: Post-operative pain and swelling are frequently observed after sinus lift procedures. The aim of the present study was a clinical evaluation of swelling and pain of two sinus lift different flaps technique measured through visual analogue scale (VAS), verbal rating scale (VRS) and infrared thermal imaging (i.e., thermography).
\end{abstract}

Methods: A randomized controlled trial was conducted in fifteen patients treated for a total of 30 sinus randomly allotted into two groups. In patients of Group I, a triangular flap was performed, while in Group II modified triangular flap without anterior release was performed. Postoperative pain was scored by VAS and inflammation was recorded by VRS at 2, 4, 6 and 14 days. The facial temperature was recorded before and after sinus augmentation, at 2, 4, 6 day intervals for the first week and at 14 day, to check the course of healing.

Results: In group I the pain intensity was recorded at 2 days after the surgery with a score of 38.67 $\pm 6.4 \mathrm{~mm}$. Swelling was higher at 2 and 4 day, and it was absent at day 6 . Facial temperature difference before and after the procedure was $3\left(4.737^{\circ} \mathrm{C} \pm 0.37\right)$. In Group II the pain was lower than in Group I $(\mathrm{p}<0,05)$. Swelling scored 2 on the first and second day, and it was reduced at day 4. After the second day the difference of temperature reduced significantly compared to day of surgery $\left(0.77^{\circ} \mathrm{C}\right)$, at 2 and 4 days no difference was registered.

Conclusion: In conclusion based on the outcome of the present study, modified triangular flap can be used with success in sinus augmentation procedures and is to be preferred to trapezoidal flap.

Trial Registration: The trial is registered at the Brazilian Clinical Trials Registry / Registro Brasileiro de Ensaios Clínicos (ReBEC) (UTN: \#U11111-1202-3138).

Key-words: trapezoidal flap, modified triangular flap, sinus augmentation, VAS, VRS, thermal infrared imaging. 
Running title: Lateral sinus floor elevation performed with two different flap designs trapezoidal and triangular.

\section{Introduction}

Post-operative pain and swelling are frequently observed after sinus lift procedures. Orofacial pain management is a challenging topic for the dental-medical profession.

The placement of dental implants in the distal edentulous maxillary area is often a problem in case of the severely resorbed maxilla and presents a complex challenge to the oral and maxillofacial surgeon.[1-5] Inadequate bone height in the lateral part of the maxilla forms a contra-indication for implant surgery, and due to the significant resorption in the posterior maxilla following teeth extraction and the pneumatization of the maxillary sinus, there is not enough bone volume to ensure primary stability of dental implants.[6-9] The goals of sinus augmentation procedures are: formation of vital bone, implant integration in newly-formed bone, long-term implant survival after functional load.[10-15] Autogenous bone is considered ideal as an augmentation material.[2]

Since there are a large number of bone substitutes available, the properties of these materials, related to biocompatibility and function in comparison to the gold standard, must be considered by surgeons in making recommendations and assisting in the patient's treatment-planning process. Various grafting materials have been used for sinus augmentation: autologous bone, mineralized and demineralized freeze-dried allografts, coralline calcium carbonate, bioglass, polylactidepolyglicolide materials, synthetic polymers, calcium sulfate, anorganic bovine bone, hydroxyapatite.[7] The sinus augmentation procedure causes a discomfort in the first to third day, although this can vary from person to person. The patients present a swelling or bruising, typically the worse swelling occurs on the third day, and then will gradually decrease. In clinical practive are difficult to evaluation pain and swelling in sinus surgery. To overcome these difficulties we used infrared thermography. The purpose of the present study was a clinical evaluation of swelling and 
pain with two different flaps for sinus lift, assessed through infrared thermal imaging (i.e., thermography) and visual analogue scale.

\section{Materials and Methods}

The protocol for this study was designed in accordance with the Helsinki Declaration (revised version of Tokyo at 2004) and Good Clinical Practice Guidelines. It was approved by the Inter Institutional Ethics Committee of Faculdade Ingá, UNINGÁ, PR, Brazil, $\mathrm{N}^{\circ}$ 72105917.5.0000.5220.

Fifteen healthy patients with non-contributory past medical history ( 9 women and 6 men, all nonsmokers, mean age 53 years, range $45-61$ years) were included in this randomized pilot study. A total of 30 sinus were randomly allotted into two groups, with 15 sinus each. All patients, that were candidates for augmentation in the posterior maxilla, were scheduled to receive fixed prosthesis or crown restorations. All patients signed a written informed consent. All patients were treated at the Department of Oral Implantology, Center for advanced studies, Dental Research Division, UNINGÁ - Cachoeiro de Itapemirim, Brazil. At the initial visit, all subjects underwent a clinical and occlusal examination. Panoramic radiographs were evaluated as well.

The inclusion criteria were: edentulous or partly edentulous with a unilateral or bilateral loss of teeth in the maxillary premolar or molar areas with a severe alveolar atrophy and a residual alveolar ridge height of between 2 and $4 \mathrm{~mm}$. The exclusion criteria were: severe illness, head and neck radiation therapy, chemotherapy, uncontrolled diabetes, uncontrolled periodontal disease, and smoking. Exclusion criteria also included facial or neck inflammatory skin diseases, carotid sinus hyperaesthesia, hyperthyroidism, and patients who rejected unilaterally as a part of post-operative treatment.

Of these patients, one patient was totally edentulous in the maxilla, and nineteen others were partially edentulous in the posterior maxilla. After a thorough oral and physical examination, 
patients were scheduled for bone reconstruction procedures, including sinus augmentation and implant insertion. Preoperatively, they were extensively informed concerning the surgical procedures and they were asked for their full cooperation during treatment.

\section{Surgical procedures}

\section{Flap design}

All patients were treated in the Outpatient Department of Oral Implantology, Center for advanced studies, Dental Research Division, UNINGÁ - Cachoeiro de Itapemirim, Brazil, and 39 implants were placed after 6 mounths of healing. All patients were evaluated for 14 days. The sites were randomly assigned to the test or control group by a computer-generated table, which was prepared using a balanced, randomly permuted sinus approach. All sinus lifts were performed by a single operator, experienced in both the trapezoidal flap (Fig. 1) and the modified triangular flap without anterior release (Figs. 2A-2B). The primary predictor variable was the flap type. There were two different flap designs used, the trapezoidal flap and the modified triangular flap. The trapezoidal flap technique was used for Group I: the incision was made on the top of the alveolar ridge horizontally with a relieving incision in the mesial and distal region. The full thickness flap was elevated with a flap elevator that must be adherent to the bone so that the periosteum remains undamaged (Fig. 1). The modified triangular flap type was used for Group II: the first part of the incision was similar to that in Group I and it extended mesially if the patient was edentulous (Figs 3A-3B), in the presence of teeth the incision was continued by a sulcular incision starting near the mesialbuccal edge of the teeth and then extended up to the midpoint of the buccal sulcus of the canine (Figs 4A-4B), with cutting the interdental papilla (Fig. 4C-4D). The flap designs were randomly assigned for each patient. Postoperative pain was scored by means of a 100-mm VAS from 0 (no pain) to 100 (worst pain imaginable) at 2, 4 and 6 day intervals for the first week and at 14 days. Pain intensity was classified into four categories: Inflammation was recorded by verbal rating scale (VRS). In this scale, swelling was classified into four categories: number 1 stands for 
the absence of inflammation, patients with an intra-oral swelling in the surgical zone scored 2, any extra-oral swelling in the surgical zone scored 3, an intense inflammation exhibited by extra-oral swelling extended beyond the surgical zone scored 4. [16] At each control the facial temperature was also measured. An independent examiner assessed the degree of swelling once daily for 14 postoperative days. All measurements were performed at the same time. The sinuses were randomly allotted into two groups, with 15 sinus each. In Group I the TZ flap was utilized and in Group II the MT flap was utilized to access the sinus area.

Prior to surgery, the patient's mouths were rinsed with a chlorhexidine digluconate solution $0.2 \%$ for 2 minutes. Local anesthesia was obtained with Articaine ${ }^{\circledR}$ (Ubistesin 4\% - Espe Dental AG Seefeld, Germany) associated with epinephrine 1:100.000. Full thickness flaps were elevated to expose the alveolar crest and the lateral wall of the maxillary sinus. Using a round bur under cold $\left(4-5^{\circ} \mathrm{C}\right)$ sterile saline irrigation, a trap door was made in the lateral sinus wall. The door was rotated inward and upward with a top hinge to a horizontal position. The sinus membrane was elevated with curettes of different shapes, until it became completely detached from the lateral and inferior wall of the sinus. The maxillary sinus was filled with sterilized bovine bone particles mixed with venous blood (Biotem Oss ${ }^{\circledR}$, Bologna, Italy). No perforation of the sinus membrane was evident in twentyfive of the cases. A small perforation was evident in five cases. Flaps were carefully sutured with Polimid 4.0 (Sweden \& Martina, Italy) in group I while in group II the distal relieving incision was left without suture as passive drains while the flap was sutured. Amoxicillin $(1 \mathrm{~g}, 2$ times per day) was prescribed for one week. Patients allergic to penicillin were prescribed Clindamycin 300 mg twice a day for 6 days. The therapy was being standardized and the dose of anti-inflammatory was homogeneous for all patients. To help reduce symptoms, analgesics medication (Ibuprofen 600mg) 2 hours following surgery and every 6 hours afterward was prescribed, to be continued for 3 days following surgery in all patients. This ensures that the medication acts prior to the local anesthetic wearing off, when it may be more difficult to control the pain. To help reduce any discomfort with sinus pressure and the surgery and to ensure adequate aeration of the surgical site, 
a nasal decongestant Mometasone Furoato Monoidrato (Rinelon spray, Msd Italia Srl) was also prescribe to reduce or eliminate the feeling of a stuffy nose 1 day before surgery and every 12 hours following surgery, to be continued for 6 days following surgery. No corticosteroids were prescribed. Taking the medication with soft foods and/or a large glass of fruit juice lessons for reduced any side effects of nausea or stomach upset. Sutures were removed two weeks after surgery. Follow-up surgical visits were scheduled at 2, 4 and 6 day intervals for the first week and at 14 , to check the course of healing. The recall program included assessments of VAS, VRS and facial temperature assessment.

\section{Temperature Measurements}

Thermal measurements were performed in a climate-controlled room (temperature: $22-24^{\circ} \mathrm{C}$, relative humidity: $50 \pm 5 \%$, and no direct ventilation on the bone).

Facial temperature of the operated side was obtained using a 14-bit digital infrared camera (FLIR SC660 QWIP, Flir Systems, Danderyd, Sweden). The acquisition parameters were: 320x240 Focal Plane Array; 8-9 $\mu \mathrm{m}$ spectral range; $0.02 \mathrm{~K}$ Noise Equivalent Temperature Differences (NETD); 50 Hz sampling rate; optics: germanium lens; f 20; and f/1.5. The camera was set 0.50 meters away from the face for maximum spatial resolution. Images were acquired at a rate of 25 per second and subsequently re-aligned using an edge-detection based method implemented in an in house software. Temperature changes in face were computed on the realigned thermal images. Thermographic data analysis was performed using FLIR QuickReport v.1.2 (FLIR Systems Inc., North Billerica, MA), which includes a tool to obtain maximum, minimum, and average temperature of a user-defined area. The facial areas of interest were malar and cheek area. 


\section{Statistical evaluation}

A power analysis was performed using clinical software for determining the number of sinus needed to achieve statistical significance for quantitative analyses of VAS, VRS and facial temperature. A calculation model was adopted for dichotomous variables (yes/no effect) by putting the effect incidence designed to discern the reasons, $85 \%$ for TZ flap and $15 \%$ for MTF flap, with alpha $=0.05$ and power $=80 \%$.

The optimal number of samples for analysis is 14 sinus augmentations.

Correlation between temperature and pain at each time was assessed by a linear regression model; and to assess the correlation of measurements throughout time, mixed effect repeated measures were performed. To evaluate the differences the VAS, VRS and facial temperature between the Groups was used one way ANOVA statistical analysis. A value of $p \leq 0.05$ was considered to be statistically significant. Data treatment and statistical analysis were done by Excel, Origin and SPSS software.

\section{Results}

\section{Group I TZ flap}

All grafted sinuses healed without complications. All augmented sinuses healed well with no occurrence of symptoms indicating possible maxillary sinusitis. Based on the VAS scores, peak pain intensity was recorded at 2 days after surgery with a score of $38.67 \pm 6.4 \mathrm{~mm}$ (Table I and Figs. 5-8A-8B). After the second day the pain intensity reduced significantly during the consecutive time intervals. Swelling was scored $3.27 \pm 0.59$ at second days and reduced significantly after four days and there was no swelling on day 6 after the surgery (Table I). Table I and and Figs 5-6 demonstrates the values of swelling and pain. 
Difference in temperature before the procedure (measurement basal) and immediately after sinus augmentation was $1.55^{\circ} \mathrm{C}$ (Table I and Fig. 7). The difference of temperature increased to two day (Fig. 8A-8B) and reduced significantly on day 6. (Table II).

\section{Group II}

MT flap

No postoperative paresthesia or signs of infection were seen during the entire healing period.

All grafted sinuses healed without complications. All augmented sinuses healed well with no occurrence of symptoms indicating possible maxillary sinusitis. The clinical healing of the surgical area was uneventful in all patients. Wound dehiscence or infection was not observed in any of the cases. The pain on the VAS scores was mild on the second and fourth day and was lower than in group I. The pain intensity was recorded at 2 days after the surgery with a score of $29.33 \pm 7.03 \mathrm{~mm}$ which is mild (Tables I-II). At four days the pain intensity reduced significantly with a score of $23.33 \pm 6.17$. Swelling scored $2.27 \pm 0.45$ on second days, $1.26 \pm 0.45$ on four days, $1 \pm 0$ on six days, on fourteen days the score was 0 (Table I). Table I shows the values of swelling and pain. The swelling was less at all times than in group I. Difference in temperature before the procedure (measurement basal) and immediately after sinus augmentation was $0.51{ }^{\circ} \mathrm{C}$ (Table II). After surgery the difference of temperature reduced significantly on day 4 on days 6 and 14 no difference was registered. (Table I and Fig. 7).

The difference of temperature increased to two day (Table II and Figs. 8C-8D) and reduced significantly on day 4 . (Table II and Fig. 7). 


\section{Statistical evaluation}

A significant differences were found in the VAS between the 2 groups at 2 days $(p=0.0004)$, at 4 days $(\mathrm{p}=0.002)$, and at 6 days $(\mathrm{p}=0.01)$ no differences were found at 14 days.

A significant difference were found in the VRS between the 2 groups at 2 days $(p=0.000018)$, at 4 days $(p=0.0008)$, and at 6 days $(p=0.009)$ no differences were found at 14 days.

A significant difference were found in the temperature between the 2 groups before and after surgery $(p=0.00013)$, at 2 days $(p=0.003)$, at 4 days $(p=0.02)$, no differences were found at 6 days $(p=0.18)$ and at 14 days.

\section{Discussion}

Sinus lift is one of the painful and discomforting treatments that determine postoperative pain and swelling. The results of this clinical study showed a significant effectiveness of the modified triangular flap for performing sinus lifts with reduction of postoperative pain and swelling. The results of this study show a statistically significant differences for pain, swelling and temperature between two types of flap were observed. This is the first study on the utilization of the modified triangular flap in sinus lift procedure. Unfortunately, most oral surgeries are accompanied by some degree of discomfort with pain and swelling. The postoperative pain evaluation in patients can be a difficult task, since it is a multidimensional experience that possesses sensory and affective components that are often hard to discriminate with the existing scores. ${ }^{17}$ For this reason a complementary observational pain measurement should be used to assess pain intensity. The most common way to assess postoperative pain in the reviewed studies has been the use of visual analog scales (VAS) and acount of the amounts of analgesics consumed. ${ }^{18}$ Swelling can be quantified in different ways. In the case of removal of impacted third molars one of the most commonly used methods is that of Amin and Laskin ${ }^{19}$, which uses suture thread grasped with two mosquito forceps to measure distances at the following reference points: from the external palpebral angle to the gonial angle on the operated side; from the lower margin of the tragus to the external angle of the 
oral commissure; and from the lower margin of the tragus to the midpoint of the chin symphysis. These assessments are difficult to apply to the evaluation pain and swelling in sinus surgery. To overcome these difficulties, we used infrared thermography. This has been used for the evaluation of temperature during implant preparation [20], treatment in diseases such as neuropathic pain, postherpetic neuralgia, whiplash injuries, inflammatory arthritis, temporomandibular joint disorders, headache, and myofascial pain syndromes [21], as well as a diagnostic guide and therapeutic tool [22], in different anatomical locations [23]. Several factors probably influence the postoperative pain and swelling. Age, sex, the duration of surgery and other environmental factors potentially affect the discomforts experienced after implant surgeries. However the duration of surgery influences post-operative pain. Patients with longer surgery experienced more pain postoperatively [24]. In the present study, the two control and test groups were uniform with respect to given factors; the patients were chosen in the same age range, the groups had approximately the same number of men and woman, the duration of the surgeries were all between 20 to 25 minutes. All the patients were prescribed a similar pharmacological therapy. In fact skin temperature is the most important method to determine the presence of mineralized tissues swelling and inflammation. The flap design is important in oral surgery. In fact, the ultimate goal in surgical sinus is not only the bone augmentation but also the preservation of periodontal tissues, using suitable surgical techniques, and the reduction of postoperative swelling. Usually, in sinus surgery, a full-thickness mucoperiosteal flap elevation with a crestal and bi-lateral oblique releasing mesial and distal incision is used.

The flap should be designed to minimize disturbance of the blood supply, and the surgical site needs to be securely covered [25]. Ideally, a mid-crestal or a slightly palatal incision should be performed, leaving at least $3 \mathrm{~mm}$ of attached tissue on the facial aspect of the incision, with anterior and posterior vertical release. These vertical incisions should be at least $5 \mathrm{~mm}$ away from the planned osteotomy (Fig. 1). A full-thickness mucoperiosteal flap is then raised and the lateral aspect of the maxillary sinus is exposed. The infraorbital foramen should be avoided during mesial 
release, precautions should be taken not to injure the neurovascular bundle during the preparation of the door and retraction of the flap. In the case of modified triangular flap the risk of injuring the infraorbital neurovascular does not exist.

The results of this clinical study showed significant effectiveness of the modified triangular flap in sinus lift procedure in reducing pain and swelling. Probably the posterior passive drains reduce swelling and pain in the use of the modified triangular flap, in fact the distal relieving incision was left without suture. This passive drain promotes the spontaneous leaking of the inflammatory exuding and reduces postoperative swelling and pain. The MT Flap cannot damage the lateral blood supply, which runs posterior to anterior. In particular, the anterior vertical incision may have a reduced blood supply. The reduced pain and swelling found in MT flaps are due to the spontaneous passive drains and to a greater blood supply. Based on the outcome of the present study, the MT flap is effective in reducing pain and swelling after sinus lifting.

In conclusion a modified triangular flap can be used with success in sinus augmentation procedures and it is to be preferred to trapezoidal flap.

\section{Conflict of Interest Statement}

No conflict of interest.

\section{Author Contribution Statement:}

Antonio Scarano, Concept/Design of study, Drafting article, surgery of the patients, approval of the submitted and final versions the paper.

Felice Lorusso, Data collection, approval of the submitted and final versions the paper.

Merla Arcangelo, Data analysis, critically revising of paper, approval of the submitted and final versions the paper.

Pablo Santos de Oliveira, Data collection, approval of the submitted and final versions the paper. 


\section{References}

1. Wallace, S. S.; Froum, S. J. Effect of maxillary sinus augmentation on the survival of endosseous dental implants. A systematic review. Ann. Periodontol. 2003, 8, 328-343, doi:10.1902/annals.2003.8.1.328.

2. Valentini, P.; Abensur, D.; Densari, D.; Graziani, J. N.; Hämmerle, C. Histological evaluation of Bio-Oss in a 2-stage sinus floor elevation and implantation procedure. A human case report. Clin. Oral Implants Res. 1998, 9, 59-64.

3. Yamamichi, N.; Itose, T.; Neiva, R.; Wang, H.-L. Long-term evaluation of implant survival in augmented sinuses: a case series. Int. J. Periodontics Restorative Dent. 2008, 28, 163-169.

4. Piattelli, M.; Favero, G. A.; Scarano, A.; Orsini, G.; Piattelli, A. Bone reactions to anorganic bovine bone (Bio-Oss) used in sinus augmentation procedures: a histologic long-term report of 20 cases in humans. Int. J. Oral Maxillofac. Implants 1999, 14, 835-840.

5. Landi, L.; Pretel, R. W.; Hakimi, N. M.; Setayesh, R. Maxillary sinus floor elevation using a combination of DFDBA and bovine-derived porous hydroxyapatite: a preliminary histologic and histomorphometric report. Int. J. Periodontics Restorative Dent. 2000, 20, 574-583.

6. Mangano, C.; Scarano, A.; Perrotti, V.; Iezzi, G.; Piattelli, A. Maxillary sinus augmentation with a porous synthetic hydroxyapatite and bovine-derived hydroxyapatite: a comparative clinical and histologic study. Int. J. Oral Maxillofac. Implants 2007, 22, 980-986.

7. Scarano, A.; Degidi, M.; Iezzi, G.; Pecora, G.; Piattelli, M.; Orsini, G.; Caputi, S.; Perrotti, V.; Mangano, C.; Piattelli, A. Maxillary sinus augmentation with different biomaterials: a comparative histologic and histomorphometric study in man. Implant Dent. 2006, 15, 197207, doi:10.1097/01.id.0000220120.54308.f3.

8. Froum, S. J.; Tarnow, D. P.; Wallace, S. S.; Rohrer, M. D.; Cho, S. C. Sinus floor elevation using anorganic bovine bone matrix (OsteoGraf/N) with and without autogenous bone: a 
clinical, histologic, radiographic, and histomorphometric analysis--Part 2 of an ongoing prospective study. Int. J. Periodontics Restorative Dent. 1998, 18, 528-543.

9. Artzi, Z.; Nemcovsky, C. E.; Dayan, D. Bovine-HA spongiosa blocks and immediate implant placement in sinus augmentation procedures. Histopathological and histomorphometric observations on different histological stainings in 10 consecutive patients. Clin. Oral Implants Res. 2002, 13, 420-427.

10. Valentini, P.; Abensur, D. Maxillary sinus floor elevation for implant placement with demineralized freeze-dried bone and bovine bone (Bio-Oss): a clinical study of 20 patients. Int. J. Periodontics Restorative Dent. 1997, 17, 232-241.

11. Valentini, P.; Abensur, D.; Wenz, B.; Peetz, M.; Schenk, R. Sinus grafting with porous bone mineral (Bio-Oss) for implant placement: a 5-year study on 15 patients. Int. J. Periodontics Restorative Dent. 2000, 20, 245-253.

12. Yildirim, M.; Spiekermann, H.; Biesterfeld, S.; Edelhoff, D. Maxillary sinus augmentation using xenogenic bone substitute material Bio-Oss in combination with venous blood. A histologic and histomorphometric study in humans. Clin. Oral Implants Res. 2000, 11, 217229.

13. Hallman, M.; Sennerby, L.; Lundgren, S. A clinical and histologic evaluation of implant integration in the posterior maxilla after sinus floor augmentation with autogenous bone, bovine hydroxyapatite, or a 20:80 mixture. Int. J. Oral Maxillofac. Implants 2002, 17, 635643.

14. Karabuda, C.; Ozdemir, O.; Tosun, T.; Anil, A.; Olgaç, V. Histological and clinical evaluation of 3 different grafting materials for sinus lifting procedure based on 8 cases. J. Periodontol. 2001, 72, 1436-1442, doi:10.1902/jop.2001.72.10.1436.

15. Miser, A. W.; Dothage, J. A.; Wesley, R. A.; Miser, J. S. The prevalence of pain in a pediatric and young adult cancer population. Pain 1987, 29, 73-83. 
16. Al-Samman, A. A.; Othman, H. A. Facial expression drawings and the full cup test: valid tools for the measurement of swelling after dental surgery. Br. J. Oral Maxillofac. Surg. 2017, 55, 22-25, doi:10.1016/j.bjoms.2016.08.015.

17. Crellin, D.; Sullivan, T. P.; Babl, F. E.; O’Sullivan, R.; Hutchinson, A. Analysis of the validation of existing behavioral pain and distress scales for use in the procedural setting. Paediatr. Anaesth. 2007, 17, 720-733, doi:10.1111/j.1460-9592.2007.02218.x.

18. UStün, Y.; Erdogan, O.; Esen, E.; Karsli, E. D. Comparison of the effects of 2 doses of methylprednisolone on pain, swelling, and trismus after third molar surgery. Oral Surg. Oral Med. Oral Pathol. Oral Radiol. Endod. 2003, 96, 535-539, doi:10.1016/S1079210403004645.

19. Amin, M. M.; Laskin, D. M. Prophylactic use of indomethacin for prevention of postsurgical complications after removal of impacted third molars. Oral Surg. Oral Med. Oral Pathol. 1983, 55, 448-451.

20. Scarano, A.; Piattelli, A.; Assenza, B.; Carinci, F.; Di Donato, L.; Romani, G. L.; Merla, A. Infrared thermographic evaluation of temperature modifications induced during implant site preparation with cylindrical versus conical drills. Clin. Implant Dent. Relat. Res. 2011, 13, 319-323, doi:10.1111/j.1708-8208.2009.00209.x.

21. Graff-Radford, S. B.; Ketelaer, M. C.; Gratt, B. M.; Solberg, W. K. Thermographic assessment of neuropathic facial pain. J. Orofac. Pain 1995, 9, 138-146.

22. Merla, A.; Mattei, P. A.; Di Donato, L.; Romani, G. L. Thermal imaging of cutaneous temperature modifications in runners during graded exercise. Ann. Biomed. Eng. 2010, 38, 158-163, doi:10.1007/s10439-009-9809-8.

23. Cardone, D.; Pinti, P.; Merla, A. Thermal Infrared Imaging-Based Computational Psychophysiology for Psychometrics. Comput. Math. Methods Med. 2015, 2015, 984353, doi: $10.1155 / 2015 / 984353$. 
24. Weisensee, W.; Scheer, M.; Müller, L.; Rothamel, D.; Kistler, F.; Bayer, G.; Jöhren, P.; Neugebauer, J. Impact of anxiety parameters on prospective and experienced pain intensity in implant surgery. Implant Dent. 2012, 21, 502-506, doi:10.1097/ID.0b013e3182703a44.

25. van den Bergh, J.; ten Bruggenkate, C.; Dish, F.; Tuinzing, D. Anatomical aspects of sinus floor elevations. Clin Oral Implant Res. 2000, 11, 256-265. 




Fig. 1) The trapezoidal flap with a relieving incision in the mesial and distal region (arrows) 


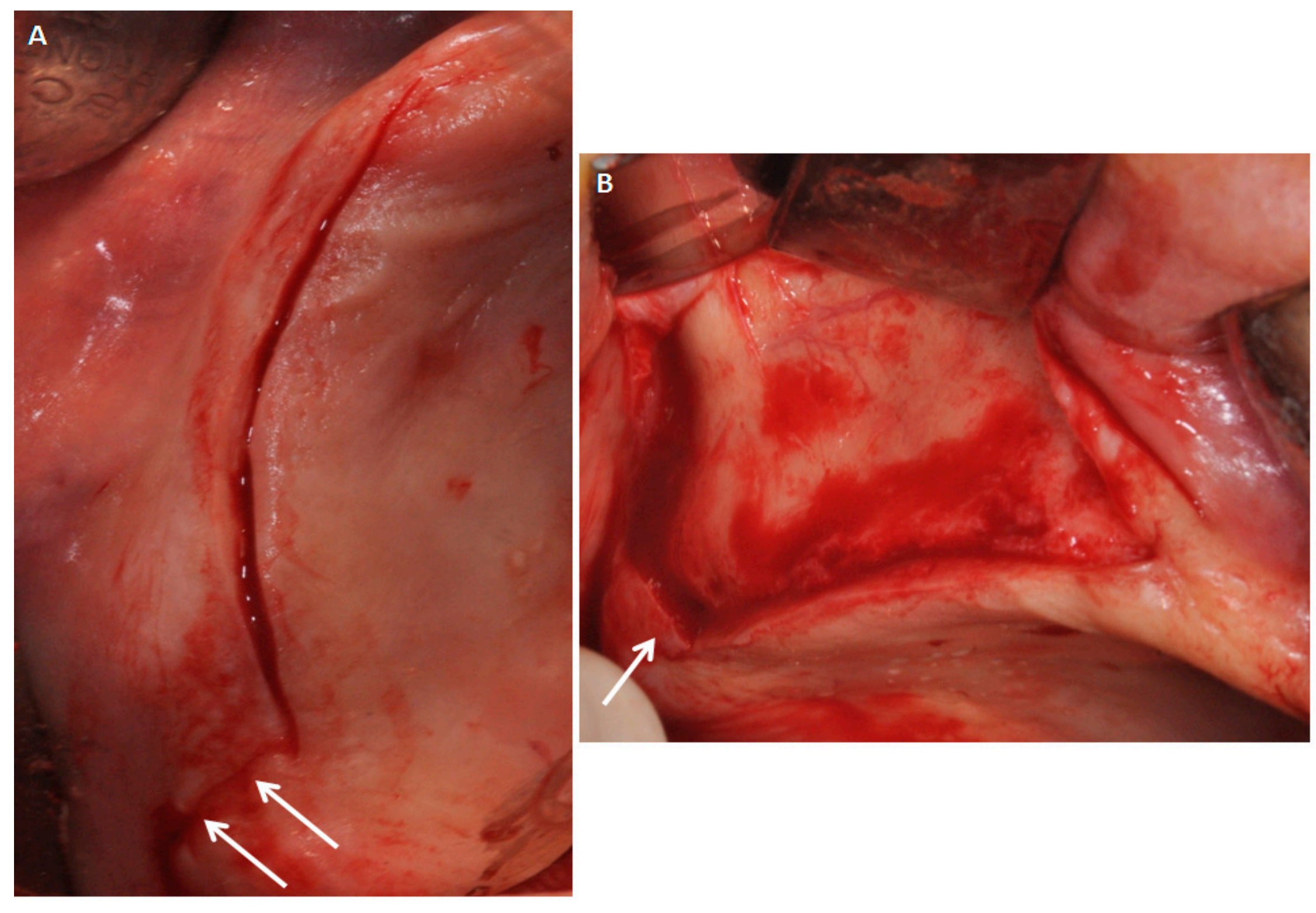

Fig. 2) A - The modified triangular flap with a relieving incision in the distal region without mesial relieving (arrows). Flap in case of edentulous patient. B - Flap elevated without difficulty. 



Fig. 3) A - Antrostomy access after dissection and elevation of the sinus membrane.

$B$ - Flaps were carefully sutured and the distal relieving incision was left without suture as passive drains (arrows) 


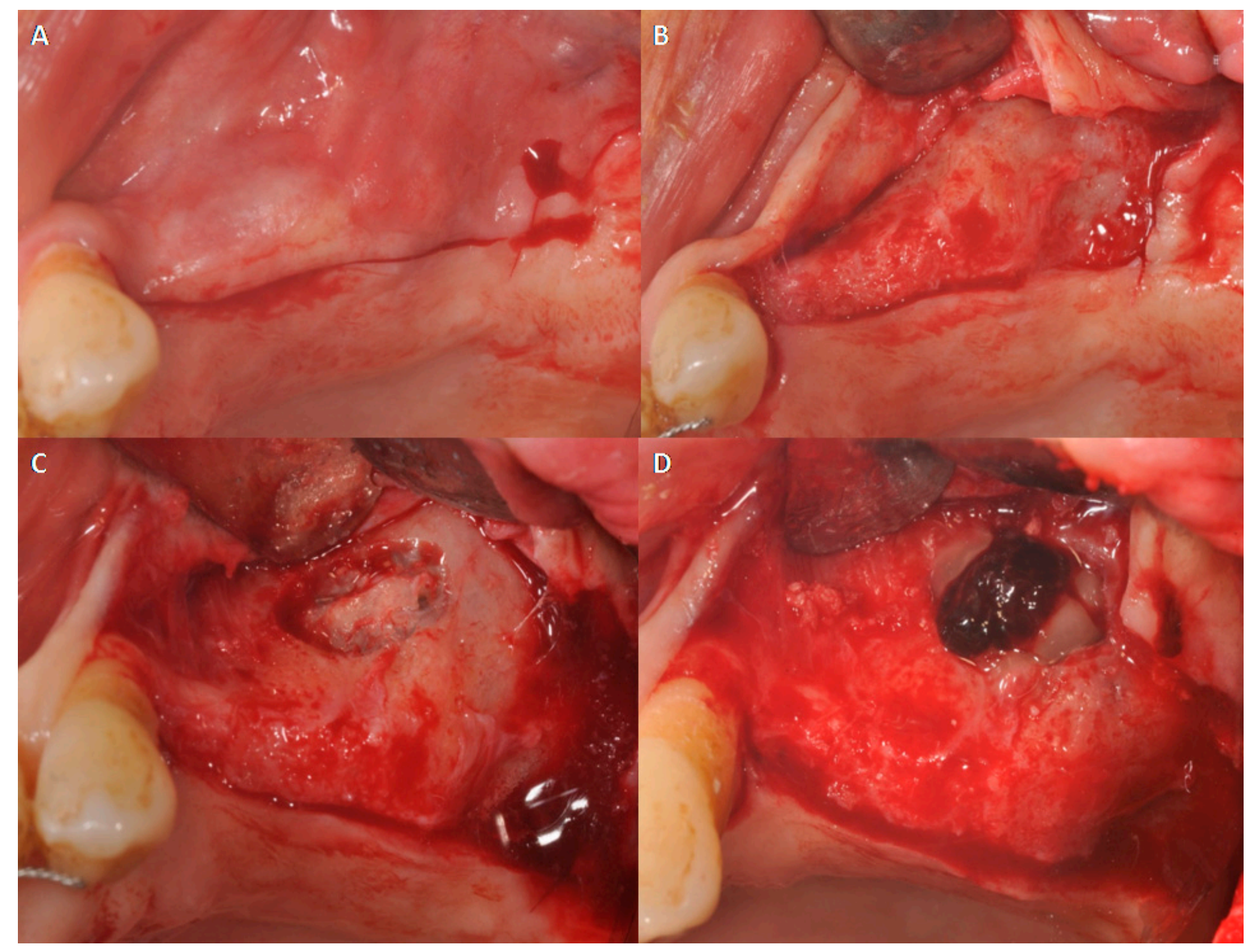

Fig. 4) A- Modified triangular technique. Flap in case of partial edentulous patient. B- The incision was continued by a sulcular incision starting near the mesialbuccal edge of the teeth. C- Flap elevated and maxillary sinus lateral wall is exposed. D- Bone window is made 
SWELLING (VRS)

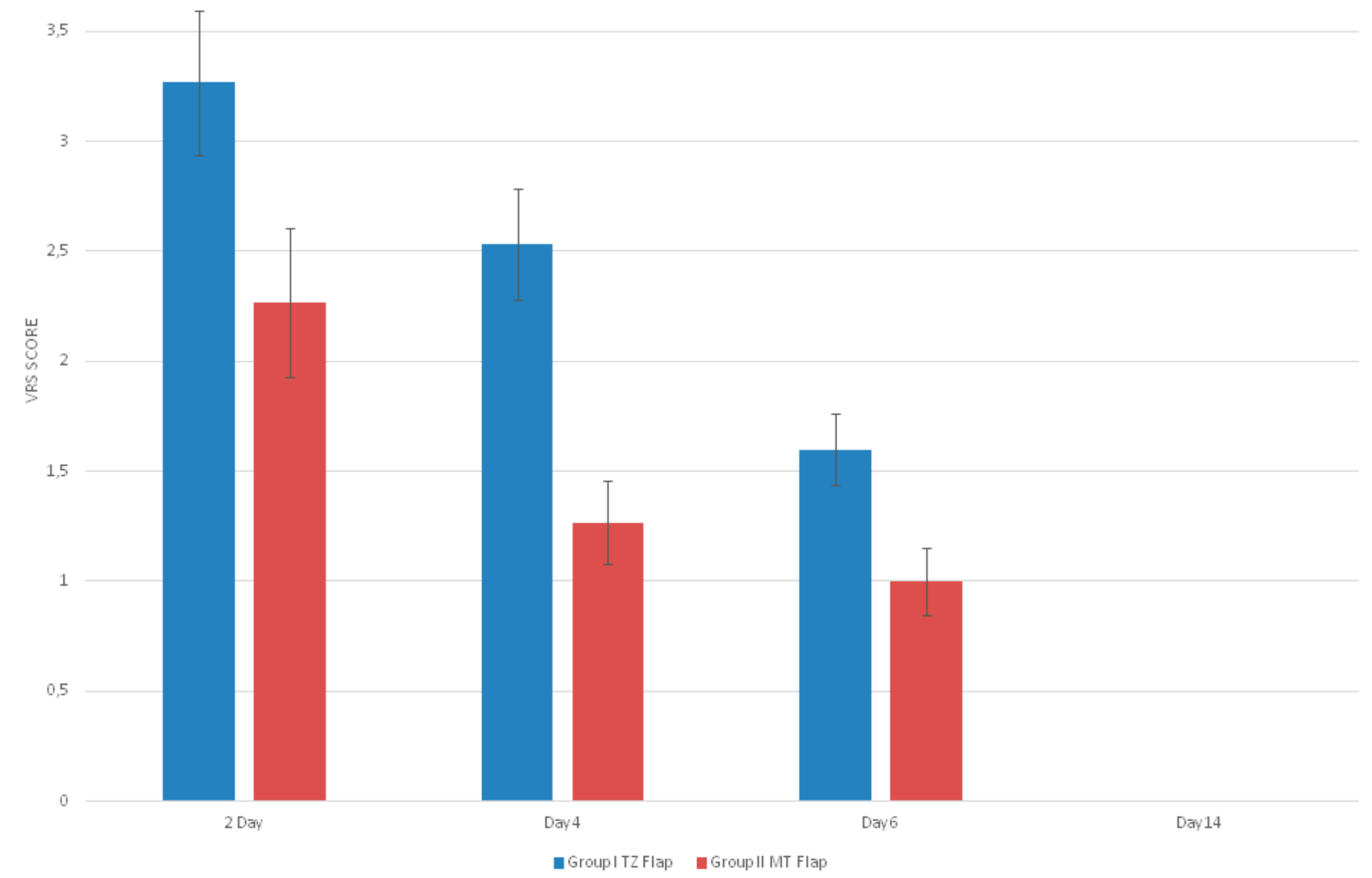

Fig. 5) Pain measurement based on the VAS scores 




Fig. 6) Swelling measurement based on the VAS scores 


\section{FACIAL TEMPERATURE $\left({ }^{\circ} \mathrm{C}\right)$}

- Group I TZ Flap a Group II MT Flap

36

35 BEFORE



AFTER

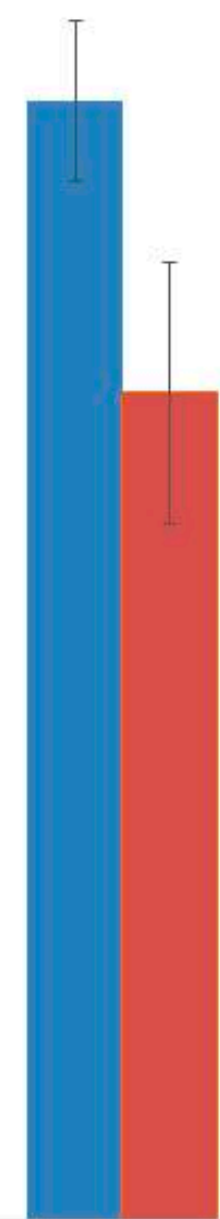

DAY 2
DAY 4

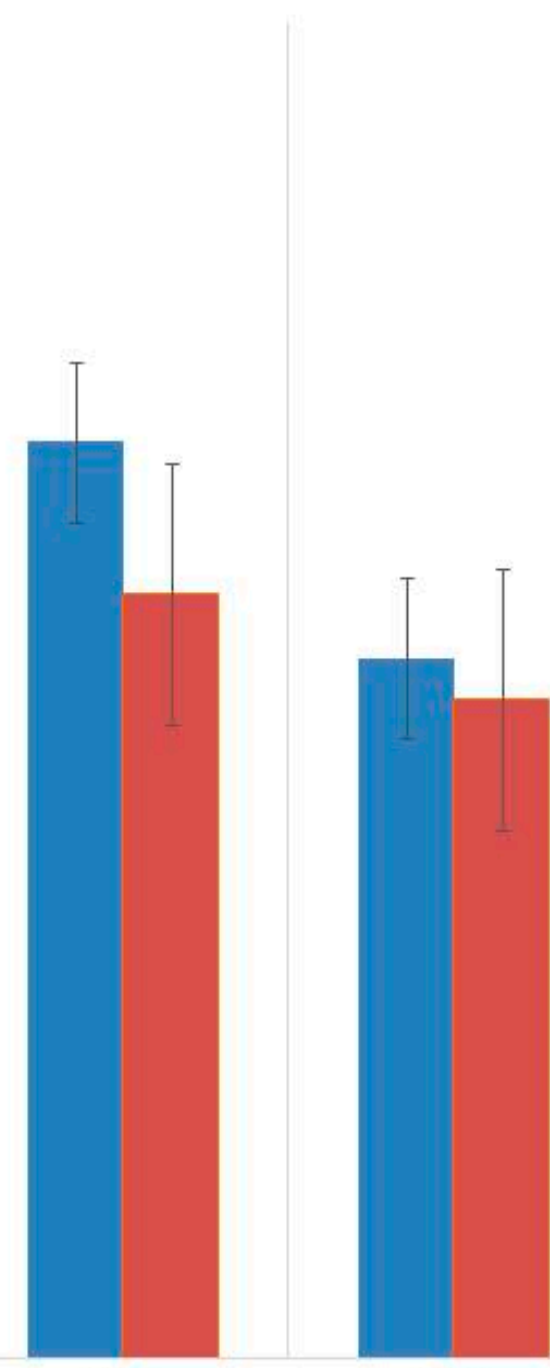

DAY 6

Fig. 7) Facial temperature measurement before the procedure and after sinus augmentation 

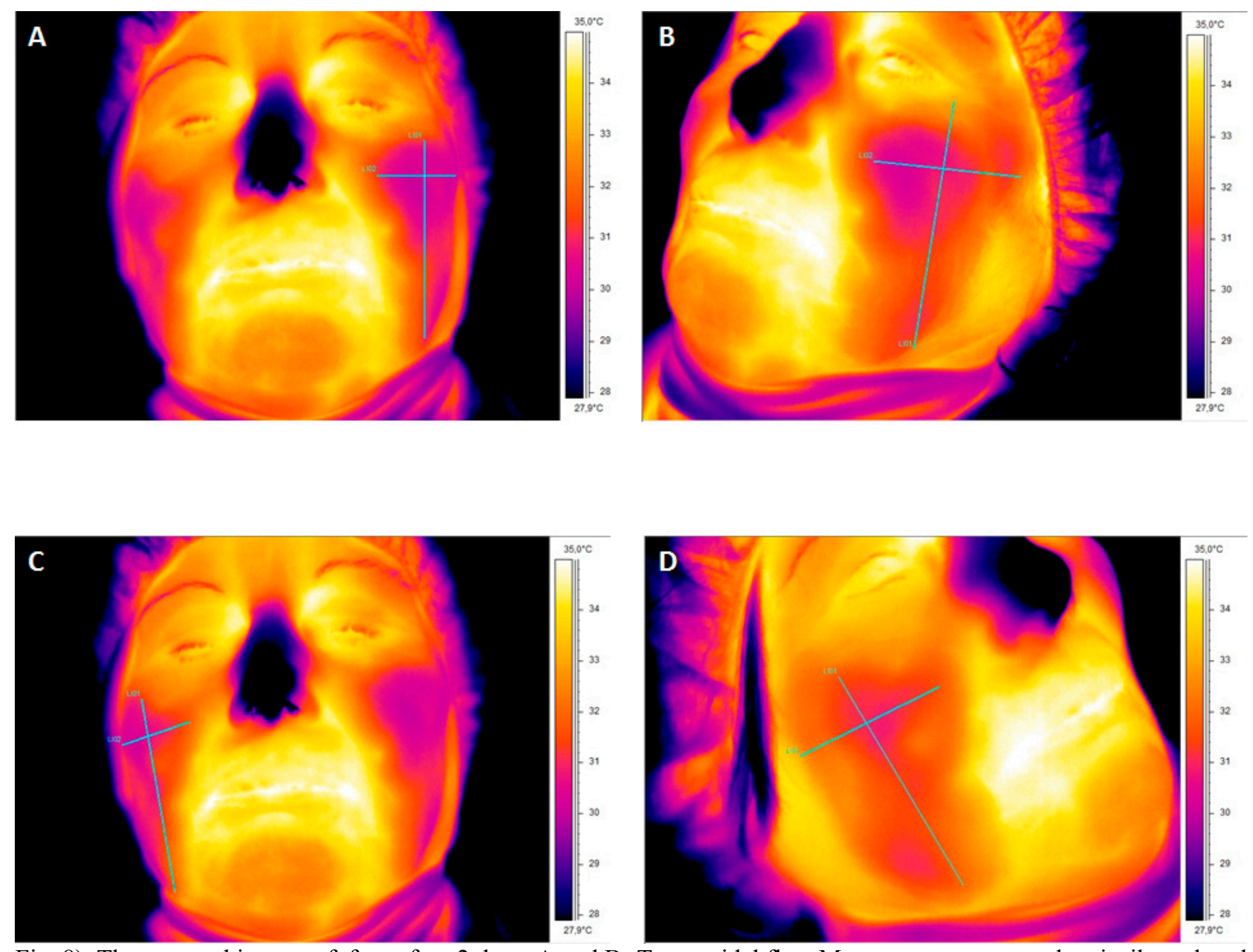

Fig. 8) Thermographic scan of face after 2 days. A and B- Trapezoidal flap. Measurements were taken ipsilateral to the side of sinus lifting. C and D- Modified triangular technique. The surface temperature is lower than the trapezoidal flap. 
Table $\mathbf{n}^{\circ} 1$

\begin{tabular}{|c|c|c|c|c|c|c|c|c|}
\hline \multirow[b]{2}{*}{ Group I TZ Flap } & \multicolumn{4}{|l|}{ PAIN (VAS) } & \multicolumn{4}{|c|}{ SWELLING (VRS) } \\
\hline & Day 2 & Day 4 & Day 6 & Day 14 & Day 2 & Day 4 & Day 6 & Day 14 \\
\hline Min & 30 & 15 & 10 & 0 & 2 & 2 & 1 & 0 \\
\hline Max & 50 & 50 & 30 & 0 & 4 & 4 & 3 & 0 \\
\hline Mean & 38.67 & 30.33 & 16.33 & 0 & 3.27 & 2.53 & 1.60 & 0 \\
\hline$S D$ & 6.40 & 9.34 & 6.11 & 0 & 0.59 & 0.74 & 0.82 & 0 \\
\hline Group II MT Flap & Day 2 & Day 4 & Day 6 & Day 14 & Day 2 & Day 4 & Day 6 & Day 14 \\
\hline Min & 20 & 10 & 0 & 0 & 2 & 1 & 0 & 0 \\
\hline Max & 40 & 30 & 20 & 0 & 3 & 2 & 1 & 0 \\
\hline Mean & 29.33 & 23.33 & 9.33 & 0 & 2.27 & 1.26 & 1 & 0 \\
\hline$S D$ & 7.03 & 6.17 & 7.98 & 0 & 0.45 & 0.45 & 0 & 0 \\
\hline
\end{tabular}

Table $\mathbf{n}^{\circ} 2$

TEMPERATURE $\left({ }^{\circ} \mathrm{C}\right)$

Group I TZFlap Before After Day 2 Day 4 Day 6 Day 14

\begin{tabular}{l|l|l|l|l|l|l|} 
Min & 34.20 & 35.2 & 35.60 & 34.9 & 34.50 & 34.3
\end{tabular}

\begin{tabular}{|l|l|l|l|l|l|l|}
\hline Max & 35.30 & 36.9 & 37.80 & 36.9 & 36.30 & 35.7 \\
\hline
\end{tabular}

\begin{tabular}{|l|l|l|l|l|l|l|} 
Mean & 34.73 & 36.28 & 37.03 & 36.11 & 35.15 & 35.13 \\
\hline
\end{tabular}

\begin{tabular}{|l|l|l|l|l|l|l|l|}
\hline SD & 0.37 & 0.44 & 0.53 & 0.55 & 0.55 & 0.52 \\
\hline
\end{tabular}

Group II MT Flap Before After Day 2 Day 4 Day 6 Day 14

\begin{tabular}{l|l|l|l|l|l|l|} 
Min & 34.20 & 34.2 & 34.10 & 34 & 34.20 & 34.3
\end{tabular}

\begin{tabular}{|l|l|l|l|l|l|l|} 
Max & 35.60 & 36.8 & 38.10 & 36.8 & 36.30 & 35.7 \\
\hline
\end{tabular}

\begin{tabular}{|l|l|l|l|l|l|l|l} 
Mean & 34.79 & 35.30 & 35.72 & 35.43 & 34.96 & 34.94 \\
\hline
\end{tabular}

\begin{tabular}{|l|l|l|l|l|l|l|l|} 
SD & 0,44 & 0.75 & 1.11 & 0.92 & 0.59 & 0.50
\end{tabular} 